\title{
Gender Differences in Stress and Coping among Adults living in Hawai i
}

\author{
Lauren A. Gentry, Jane J. Chung, Nandar Aung, Stefan Keller, \\ Katie M. Heinrich, \& Jay E. Maddock \\ University of Hawaii at Manoa School of Medicine
}

\begin{abstract}
Background and purpose. Stress has been recognized as a public health problem. However, little research has been done on gender differences in sources of stress and coping strategies in Hawaii. In this study, we hypothesized that: 1) women will report higher levels of stress than men; 2) women will report being stressed by family and health related stressors while men will report stress related to finances and work-related issues; 3) women will report using adaptive coping strategies more frequently while men will report using maladaptive and avoidance strategies more frequently; 4) there will be no gender differences in the readiness to use stress management strategies. Method. A statewide cross-sectional telephone-survey of 1518 participants was conducted during the spring and summer of 2006. Results. Women reported higher overall perceived stress levels, but there was no difference in the experienced social stressors and health stressors between genders. Men perceived more stress from personal factors. There were no gender differences in the perceived ability to cope with stress. However, women were more likely to use adaptive coping strategies, whereas men were more likely to use maladaptive and avoidance coping strategies. There were no significant gender differences in stages of change for stress management. Conclusion. Based on this study, interventions can be developed to help people better cope with stress. Interventions for women may focus on increasing the use of adaptive strategies such as praying and talking to friends and family, while interventions for men may introduce the use of adaptive coping strategies such as exercise and actively fighting causes of stress. This study shows that gender differences in stress levels and coping in Hawaii are similar to previous studies conducted on the mainland. More research into specific stressors and coping strategies may help tailor interventions that are more effective and comprehensive.
\end{abstract}

(C) 2007 Californian Journal of Health Promotion. All rights reserved. Keywords: gender differences, psychological, stress, coping, Hawaii

\section{Gender Differences in Stress and Coping among Adults Living in Hawaii}

Stress is a natural physiological mechanism that protects humans from danger. When stress occurs, the human body prepares for quick action by releasing hormones that increase alertness and focus (National Institutes of Health [NIH], 2007). However, if the source of stress does not disappear, stress hormones can persist in the body. Continual exposure to stress hormones has been linked to a wide range of physical and psychological illnesses, such as obesity,gastrointestinal disorders, cardiovascular disorders, skin disorders, anxiety attacks, and depression (Everly \& Lating, 2002; NIH, 2007; Weidner, 2000). Although the importance of stress as a public health issue has been widely recognized, many early studies of stress failed to examine the effect of gender in their data analysis or were conducted with primarily all male samples (Greenglass, 1995; Makosky, 1980).

\section{Gender Differences in Stress}

Recent studies have begun to recognize the importance of gender's influence on stress and have consistently revealed that women report higher levels of chronic and daily stressors than men (Hogan, Carlson, \& Dua, 2002; Ptacek, Smith, \& Zanas, 1992; Tamres, Janicki \& Helgeson, 2002). Using a modified version of Wheaton's chronic stress inventory, 
McDonough and Walters (2001) found that women's distress scores were $23 \%$ higher than men's. Utilizing the Life Event Stressful Success Questionnaire (LESSQ), Matud (2004) asked a sample of 1,566 women and 1,250 men between the ages of 18 and 65 for the number of major life events and changes within the previous two years. Women reported being significantly more stressed than men, even when controlling for the number of life events and changes. In addition, women were also more likely to feel that the major life events were less within their control.

\section{Sources of Stress}

Growing evidence suggests that women and men are stressed by different types of situations. Men were more likely to list finances and workrelated events as sources of their stress (stressors), whereas women were more likely to list family and health-related events (Matud, 2004; McDonough \& Walters, 2001). For example, Harriman (1983) found that the birth of a first child created significantly more stress among women than men. Tytherleigh, Jacobs, Webb, Ricketts, and Cooper (2007) also found that women and men experience stress differently in the workplace. Men seem to be more stressed by financial issues, such as worries about pay and benefits. In addition, working long hours impacted women and men differently. Women who work more than 60 hours a week had less perceived control and report higher levels of stress than women who worked fewer hours. However, men who worked more than 60 hours reported increased levels of control (Tytherliegh et al. 2007).

\section{Coping Strategies}

Being able to effectively cope with stress can be the first step to preventing psychological distress and development of a serious illness. Talking with friends and family, exercising, praying, or actively addressing the causes of stress have been identified as adaptive coping strategies that may enhance overall well-being (AndrePetersson, Hedblad, Janzon, \& Ostergren, 2006; Tyler \& Ellison, 1994; Wang \& Patten, 2002). On the other hand, maladaptive and avoidance strategies, such as drinking or denying the existence of the stressful situation, have been found to be associated with depression (Gore-
Felton, Koopman, Spiegel, Vosvick, Brodino, \& Winningham, 2006). Significant gender differences have been found for stress coping strategies (Matheny, Ashby \& Cupp. 2005). In a meta-analytic review of gender differences in coping, Tamres, Janicki, and Helgeson (2002) found that women tended to use social support and help-seeking behaviors to cope with stress, which may be protective factors against the incidence of depression and anxiety disorders (Plaisier, de Bruijn, de Graaf, ten Have, Beekman, \& Penninx, 2007; Torkelson \& Muhonen, 2004). Lindquist, Beilin, and Knuiman (1997) found that men were more likely to use maladaptive coping strategies, such as consuming alcohol and following unhealthy eating patterns. Furthermore, McDonough and Walters (2001) also found that men were five times more likely to consume more than 14 servings of alcoholic beverages during a week.

\section{Transtheoretical Model of Change Applied to Stress Management}

The Transtheoretical model (TTM) suggests that change in health behavior progresses in five successive stages: precontemplation, contemplation, preparation, action, and maintenance (Prochaska \& Velicer, 1997). This model has been successfully applied to various health behaviors such as smoking cessation, healthy eating habits, and adoption of physical activity. When exploring gender differences in the readiness for behavior change across different health behaviors, O'Hea, Wood, and Brantley (2003) found significant gender differences in stages of readiness for change in smoking and exercise behaviors. They found that in smoking, more females $(22.8 \%)$ than males $(9.7 \%)$ were in pre-contemplation stage of change. By contrast, there were more males $(51.4 \%)$ in maintenance stage of change than females $(37.1 \%)$. Significant gender difference also existed in exercise behaviors with more males in action and maintenance stages and more females in contemplation and preparation stages.

Although inadequate or insufficient use of coping strategies to deal with daily stress can be seen as a health risk behavior, not much is known about individuals' readiness to change 
this behavior. Only very few studies have applied the TTM in the context of stress management. While stress management includes a broader range of concepts than coping strategies, the results from these studies are the only ones that address the topic from a behavior change perspective. Evers, Prochaska, Johnson, Mauriello, Padula, and Prochaska (2006) found a stress-management intervention based on TTM to be effective on a population level, with over $60 \%$ of individuals in the treatment group moving from pre-contemplation stage to the action and the maintenance stages compared to $40 \%$ in the control group. Moreover, those in the treatment group were more likely to maintain their behavior at the 18-month follow-up. However, gender differences were not examined in this study. Keller, Kaluza, Baum and Basler (2002) found no gender differences in the readiness for stress management in a sample of 866 patients participating in a health check-up in Germany. Padlina, Aubert, Gehring, MartinDiener, and Somaini (2001) in Switzerland included gender as a variable but found no gender differences in stages of change in stress management. Their results showed that about one third of participants appeared to be coping successfully with stress, one third intend to do so, while one third appeared to have no intention to manage their stress more effectively. They also found that those in the maintenance stage reported significantly less symptoms of illness than others, illustrating the urgent need for the development of effective stress management intervention programs.

\section{Study Focus}

More population-based research is needed to fully understand how gender impacts experiences of stress. Previous studies have mostly used small samples from specific demographics, such as college students, employees, and athletes (Hanna, 1998; Hogan, Carlson, \& Dua, 2002; Tamres, Janicki, \& Helgeson, 2002). This study will evaluate these issues in a sample that is representative for the population of the state of Hawaii. Hawaii offers a unique socio-cultural environment and a number of specific stressors and coping strategies which make it essential that basic data about stress concepts in the population are researched. For example, living in paradise comes with a high cost of living. According to the Hawaii State Government Department of Business, Economic Development, \& Tourism (2004), a family of four living in Honolulu needs to earn $\$ 111,695$ to maintain a lifestyle similar to a comparable family earning $\$ 72,000$ in the continental United States. Financial issues may be a large source of stress for many women and men living in Hawaii. Hawaii is also one of the most ethnically diverse states in the United States, with Asian and Pacific Islanders making up $50.5 \%$ of the state's population, compared to $4.4 \%$ of the national average (US Census Bureau, 2005). Asian and Pacific Island cultures tend to be family- and community-oriented, which may cause additional stress in terms of worries about family and friends for people living in a collectivistic culture (Kim, Yang, \& Hwang, 2006).

More population-based research is needed to fully understand how gender impacts experiences of stress. Previous studies have mostly used small samples from specific demographics, such as college students, employees, and athletes (Hanna, 1998; Hogan, Carlson, \& Dua, 2002; Tamres, Janicki, \& Helgeson, 2002). The goal of this study is to evaluate how men and women living in Hawaii experience and cope with stress. Based on the findings of previous studies, our hypotheses are 1) women will report higher levels of stress than men 2) women will report being stressed by family and health related stressors while men will report stress related to finances and workrelated issues 3) women will report using adaptive coping strategies more frequently while men will report using maladaptive and avoidance strategies more frequently, 4) there will be no gender differences in the TTM stages of change, i.e. in the readiness for consistent stress management.

\section{Method}

\section{Design}

A cross-sectional random-digit-dialing telephone survey that collected data from a stratified sample as a part of the Healthy Hawaii Initiative (HHI). A total of 88,229 phone calls were made using a computer assisted telephone 
interviewing system (CATI) during Spring and Summer 2006. To avoid selection bias, the household member over age 18 who had the last birthday was asked to answer the survey questions over the phone. Verbal consent was acquired by the surveyor before the survey was conducted, and participants were thanked for their time and participation at the end of the survey. A total of 4,603 (5.2\%) participants completed the telephone interviews, with 4,594 meeting all of the inclusion criteria. The mean time for a telephone interview was 23 minutes. The survey was approved by the University of Hawaii at Manoa institutional review board.

\section{Survey}

The major focuses of the HHI campaign was to measure attitudes and behaviors for nutrition, physical activity, and tobacco use to ultimately enhance healthy behaviors in the state of Hawaii One third of the sample were randomly selected to receive a subset of 26 questions addressing overall perceived stress level, potential stressors, perceived effectiveness of stress coping, relevant coping strategies, and readiness for consistent stress management practice.

The participants were asked "Currently, how stressful would you say your life is?" to examine perceived stress level with a five point interval scale ranging from "not at all stressful" to "extremely stressful". Participants were also asked which of the following areas caused stress in their everyday lives: work, unemployment, own health, health of family or friends, general worries about the future, family conflicts, conflicts with friends or at work, finances, living situation, traffic, crime, drug-related issues, and discrimination. The answer format was a four point interval scale ranging from "no" to "yes, a lot"

For perceived coping effectiveness, participants were asked "Overall, how well do you manage the stress in your daily life?". Answers ranged from "not well at all" to "extremely well" on a five point interval scale. Participants also indicated the frequency with which they used selected strategies to handle stressful situations in their life. These coping strategies include exercising, eating, drinking alcohol, smoking, taking drugs, talking to friends or family, praying, trying to ignore the problem, distracting oneself, and actively fighting the causes of stress. Answers were given on a five point interval scale ranging from "never" to "very often".

Finally, participants were categorized into one of the five TTM stages of change based on their readiness to consistently use stress management strategies. The stage-defining answers were "No, and I do not intend to do so in the next six months" (precontemplation stage), "No, but I intend to do so in the next six month" (contemplation stage), "No, but I intend to do so in the next 30 days" (preparation stage), "Yes, for less than six months" (action stage), "Yes, for more than six months" (maintenance stage).

\section{Statistical Analysis}

The factor structure of the items describing potential stressors and coping strategies were analyzed using principal component analysis with varimax rotation. Factors with eigenvalues greater than one were retained and analyzed as subscales. The scale reliability was determined using Cronbach's alpha. Inter-scale correlations were determined using Pearson product-moment correlation coefficients. Gender differences were calculated by one-way ANOVAS. Effect sizes for the differences were calculated as $\eta 2$ with $>$ .01 considered a small effect, $>.06$ a medium effect and $>.14$ a large effect (Cohen, 1988). For this study, the dependent variables were the participants' current perceived stress level, causes of stress (stressors), the perceived coping effectiveness, the frequency of using selected coping strategies, and the stages of change to consistently manage use stress management strategies. The main independent variable was gender. Statistical analyses were performed with the SPSS 15.0 package (SPSS Inc., 2006).

\section{Results}

\section{Sample}

Overall, the sample was representative of Hawaii's population. Table 1 shows the demographics of the study participants. Compared to the 2005 US inter-censual estimates for the state of Hawaii, women $(55.3 \%$ vs. $50.1 \%)$ and Whites (33.3\% vs. $26.8 \%$ ) were 
slightly overrepresented in our sample (US Census Bureau, 2007). The average number of persons per household in the sample (2.89) was similar to that in the census data. However, the median income of the study sample $(\$ 60,000)$ was higher than that in the census data $(\$ 48,274)$ (US Census Bureau, 2007).

Table 1

Sample Demographics $(\mathrm{n}=1518)$

\begin{tabular}{|l|c|}
\hline \multicolumn{1}{|c|}{ Demographic Variables } & Statistic \\
\hline Gender \% & \\
\hline Male & 44.7 \\
\hline Female & 55.3 \\
\hline Race/ethnicity \% & \\
\hline Caucasian & 33.3 \\
\hline Hawaiian & 17.7 \\
\hline Chinese & 4.1 \\
\hline Filipino & 10.7 \\
\hline Japanese & 21.2 \\
\hline Other & 13.0 \\
\hline Marital Status \% & \\
\hline Married & 57.0 \\
\hline Widowed & 7.2 \\
\hline Separated/Divorced & 12.2 \\
\hline Never Married & 20.5 \\
\hline Not married but living with partner & 3.1 \\
\hline No. in household (mean (SD)) & $2.89(1.34)$ \\
\hline Age years (mean (SD)) & $50.61(16.94)$ \\
\hline Education years (mean (SD)) & $14.67(3.10)$ \\
\hline English spoken at home \% & 92.9 \\
\hline Income (median) & $\$ 60,000$ \\
\hline BMI (mean (SD)) & $26.37(6.26)$ \\
\hline Health \% & 29.0 \\
\hline Very Good Health & 37.3 \\
\hline Good Health & \\
\hline
\end{tabular}

Principal Component Analysis of Stressor and Coping Strategy Items

A total of $n=1,440$ respondents $(95 \%)$ provided complete data and were included in the principal component analysis of stressor items. The analysis resulted in three factors with eigenvalues $>1$ (see Table 2) which were labeled personal stressors $(\mathrm{n}=7)$, social stressors $(n=2)$, and health stressors $(n=2)$. These three factors accounted for $52.7 \%$ of the total variance. The reliability of the subscales was good to satisfactory based on Cronbach's alpha (see Table 2). The subscale intercorrelations were medium to high. Personal stressors correlated with social stressors at $\mathrm{r}=$ .55 and with health stressors at $r=.35$; social stressors and health stressors correlated with $\mathrm{r}=$ .45 .

Principal components analysis was then used to analyze the structure of relevant coping strategies. 
Table 2

Factor analysis and subscale reliability indicators for stressors

\begin{tabular}{|c|c|c|c|c|}
\hline \multirow[b]{2}{*}{ Single item } & \multirow[b]{2}{*}{ Mean (SD) } & F1 & F2 & F3 \\
\hline & & $\begin{array}{l}\text { Personal } \\
\text { stressors }\end{array}$ & $\begin{array}{c}\text { Social } \\
\text { stressors }\end{array}$ & $\begin{array}{c}\text { Health } \\
\text { stressors }\end{array}$ \\
\hline Finances & $1.99(1.09)$ & .74 & & \\
\hline Conflict with friends or at work & $1.48(0.84)$ & .67 & & \\
\hline Living situation & $1.55(0.90)$ & .65 & & \\
\hline Work & $2.00(1.13)$ & .61 & & \\
\hline Family conflict & $1.71(0.98)$ & .56 & & \\
\hline General worries about the future & $1.97(1.04)$ & .55 & & \\
\hline Unemployment & $1.32(0.82)$ & .50 & & \\
\hline Drug related issues & $1.71(1.04)$ & & .85 & \\
\hline Crime & $1.76(0.99)$ & & .82 & \\
\hline Discrimination & $1.47(0.87)$ & & .72 & \\
\hline Traffic & $1.88(1.08)$ & & .54 & \\
\hline Health of family or friends & $2.07(1.08)$ & & & .80 \\
\hline Your health & $1.82(1.02)$ & & & .77 \\
\hline Explained variance & & $33.4 \%$ & $11.6 \%$ & $7.8 \%$ \\
\hline Cronbach's alpha & & .77 & .76 & .59 \\
\hline
\end{tabular}

Table 3 shows the results for coping strategies. Three main factors with eigenvalues $>1$ were identified. They were labeled maladaptive $(\mathrm{n}=$ $4)$, adaptive $(n=4)$, and avoiding strategies $(n=$ 2). These three factors accounted for $51.3 \%$ of the total variance. The subscale reliability coefficients (Cronbach's alpha) for the scales were satisfactory for the maladaptive $(\alpha=.60)$ and avoiding $(\alpha=.65)$ strategies and low for the adaptive strategies $(\alpha=.50)$. The exclusion of items with low item-scale correlations did not improve the scale reliability. The subscale intercorrelations were small. Maladaptive coping correlated with adaptive coping at $r=.13$ and with avoiding at $\mathrm{r}=.29$; adaptive coping and avoiding correlated with $\mathrm{r}=.27$.

\section{Gender Differences}

Using the new factors for causes of stress and coping strategies, the data were analyzed to examine differences between genders. Table 4 shows that there was a significant difference between the genders, with women reporting higher perceived stress than men. Men reported significantly more stress from personal stressors. The effect size $(\eta 2)$ indicated that this difference was very small. No significant gender differences were found for the subscales representing social stressors and health stressors. For both men and women, the subscale 'personal stressors' was seemed significantly correlated with the perceived stress level( $\mathrm{r}=.333$ for men, $r=.439$ for women). The respective correlations were substantially smaller for perceived stress level and social stress $(r=.101$ for men, $r=.160$ for women) and health stressors $(r=.243$ for men, $r=.374$ for women).

On the level of single items, the perceived stress level correlated the highest with stress from work (men: $\mathrm{r}=.377$, women: $\mathrm{r}=.404$ ), finances (men: $r=.239$, women: $r=.357$ ), health of friends or family (men: $r=.294$, women: $r=$ .374), and general worries about the future (men: $r=.240$, women: $r=.329$ ). Single items that had the lowest correlation with total perceived 
stress level for both genders were discrimination (men: $\mathrm{r}=.110$, women: $\mathrm{r}=.090$ ), drug-related issues (men: $r=.030$, women: $r=.036$ ), and unemployment (men: $\mathrm{r}=.064$, women: $\mathrm{r}=$ $.104)$.

Table 3

Factor Analysis for Coping Strategies $(n=1,422)$

\begin{tabular}{|l|c|c|c|c|}
\hline \multirow{2}{*}{ Single item } & Mean (SD) & F1 & F2 & F3 \\
\cline { 3 - 5 } & & $\begin{array}{c}\text { Maladaptive } \\
\text { factor }\end{array}$ & $\begin{array}{c}\text { Adaptive } \\
\text { factor }\end{array}$ & $\begin{array}{c}\text { Avoiding } \\
\text { factor }\end{array}$ \\
\hline Drinking alcohol & $1.54(1.00)$ & .80 & & \\
\hline Smoking & $1.40(1.00)$ & .69 & & \\
\hline Taking drugs & $1.14(0.57)$ & .68 & & \\
\hline Eating & $2.18(1.24)$ & .40 & & \\
\hline & & & & .70 \\
\hline Talking to friends and family & $3.22(1.29)$ & & .66 & \\
\hline Exercise & $3.04(1.37)$ & & .60 & \\
\hline Praying & $3.29(1.48)$ & & .48 & \\
\hline Actively fighting causes of stress & $2.67(1.33)$ & & & \\
\hline & & & & .85 \\
\hline Ignore problems & $2.18(1.26)$ & & & \\
\hline Distracting yourself & $2.47(1.26)$ & & & \\
\hline & & & & \\
\hline Explained variance & & $24.2 \%$ & $16.1 \%$ & $11.0 \%$ \\
\hline Cronbach's Alpha & & .60 & .50 & .65 \\
\hline
\end{tabular}

Table 4

Gender differences in perceived stress and stressor subscales

\begin{tabular}{|l|c|c|c|}
\hline & Male & Female & \multirow{2}{*}{ ANOVA } \\
\cline { 2 - 3 } & Mean (SD) & Mean (SD) & \\
\hline Perceived coping effectiveness & $2.20(1.08)$ & $2.34(1.14)$ & $\mathrm{F}(1,1503)=5.85, \mathrm{p}<.05, \eta^{2}=.004$ \\
\hline Adaptive coping strategies & $1.75(0.61)$ & $1.67(0.59)$ & $\mathrm{F}(1,1484)=6.14, \mathrm{p}<.05, \eta^{2}=.004$ \\
\hline Maladaptive coping strategies & $1.73(0.79)$ & $1.69(0.74)$ & $\mathrm{F}(1,1485)=1.11, \mathrm{~ns}$ \\
\hline Avoidance coping strategies & $1.90(0.89)$ & $1.98(0.89)$ & $\mathrm{F}(1,1495)=2.90, \mathrm{~ns}$ \\
\hline
\end{tabular}

${ }^{1}$ single item

Table 5 shows that there was no significant difference between genders in overall perceived coping effectiveness. However, there was a significant difference between genders when looking at the subscales representing different coping strategies. Women were significantly more likely to use adaptive coping strategies, while men were significantly more likely to use maladaptive coping strategies or avoid the stressor. Overall effect sizes for gender differences were small or very small. 
Table 5

Gender differences in coping strategies subscales

\begin{tabular}{|l|c|c|c|}
\hline & Male & Female & \multirow{2}{*}{ ANOVA } \\
\cline { 2 - 3 } & Mean (SD) & Mean (SD) & \\
\hline Perceived coping effectiveness & $3.48(1.03)$ & $3.46(0.99)$ & $\mathrm{F}(1,1487)=0.13, \mathrm{~ns}$ \\
\hline Adaptive coping strategies & $2.91(0.86)$ & $3.17(0.85)$ & $\mathrm{F}(1,1436)=33.21, \mathrm{p}<.001, \eta^{2}=.023$ \\
\hline Maladaptive coping strategies & $1.60(0.71)$ & $1.52(0.57)$ & $\mathrm{F}(1,1474)=5.29, \mathrm{p}<.05, \eta^{2}=.004$ \\
\hline Avoidance coping strategies & $2.39(1.10)$ & $2.27(1.06)$ & $\mathrm{F}(1,1478)=4.04, \mathrm{p}<.05, \eta^{2}=.003$ \\
\hline
\end{tabular}

${ }^{\top}$ single item

Figure 1 shows the means and standard deviations for each single stressor item. This bar graph shows that work, finances, health of family or friends, and general worries about the future cause the most amount of stress for both genders. Unemployment, living situation and conflict with friends or at work cause the least amount of stress for both genders. Men have significantly more stress over work, unemployment, living situation and conflict with friends or at work. Women experience more stress from health of family or friends. There were no significant differences between men and women for stressors such as finances, family conflict, general worries about the future, drugrelated issues, crime, discrimination, traffic and personal health.

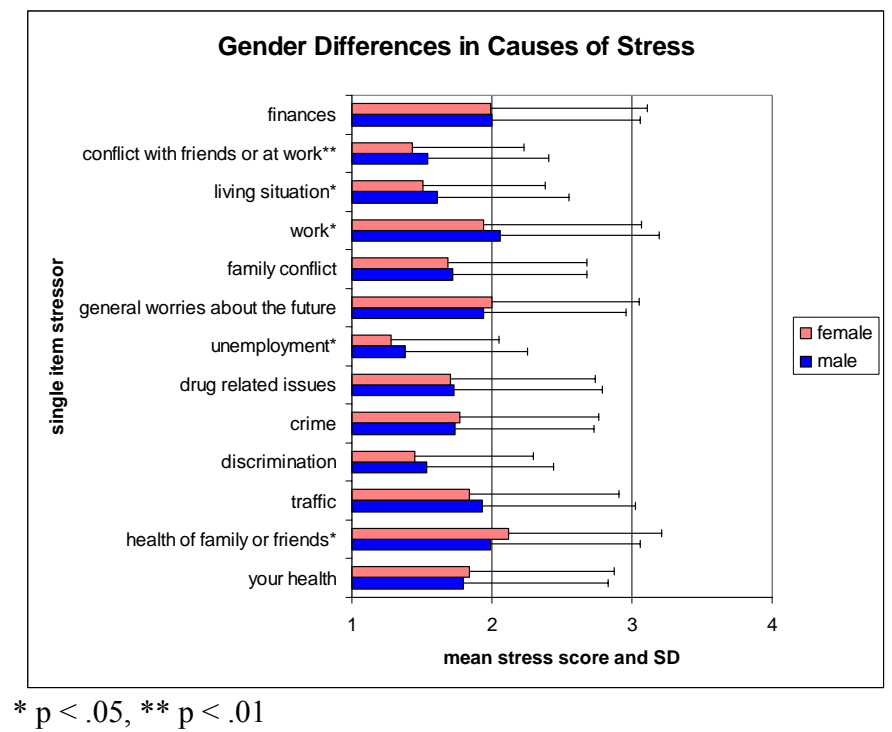

Figure 1

Gender Differences in Causes of Stress

Figure 2 shows the means and standard deviations for each single coping strategy item. Praying, exercise and talking to friends and family were the most used coping strategies.
Taking drugs, smoking and drinking alcohol were the least used coping strategies. Women were significantly more likely than men to pray, eat or talk to friends and family to cope with 
stress. Men were significantly more likely to drink alcohol, smoke, take drugs and ignore the problem to cope with stress. There were no significant differences in men and women for coping by exercising, actively fighting causes of stress or distracting.

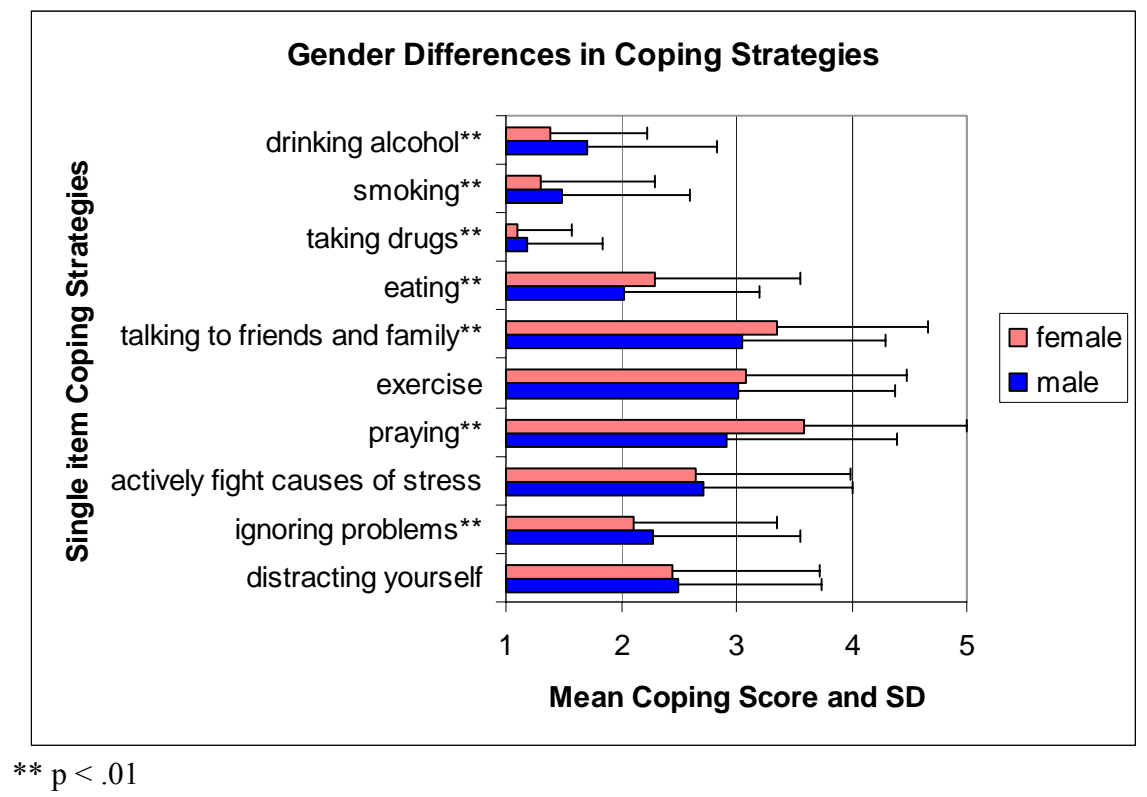

Figure 2

Gender Differences in Coping Strategies

Table 6 shows the distribution of men and women across the stages of change for stress management. The majority of women and men were in the maintenance stage. Differences in stage distribution were not statistically different between genders. This was also true when the analysis was limited to individuals who reported at least moderate perceived stress. Finally, table 6 also shows the means and standard deviations for perceives stress level and perceived coping effectiveness across the stages of change. Results for men and women were combined because two-way analyses of variance did not result in a significant main effect for gender nor in significant gender $\mathrm{X}$ stage interactions. However, there were significant main effects for stage in the perceived stress level $(F(4,1346)=$ 5.76, $\mathrm{p}<.001, \eta 2=.017)$. Post hoc analysis confirmed a significant difference in perceived stress between those in precontemplation stage and those in the maintenance stage (Tukey, p <
$.05)$. The main effect stage was also significant for perceived coping effectiveness $(F(4,1346)=$ 12.82, $\mathrm{p}<.001, \eta 2=.037)$. Individuals in the preparation and maintenance stages had higher scores than those in all other stages; additionally, individuals in the action stage had higher scores than those in the preparation stage (Tukey, $\mathrm{p}<.05$ ).

\section{Discussion}

Although the magnitude of gender differences was small, the results of our study suggest the existence of significant gender differences in experiences of stress. Consistent with the results of previous studies (e.g., Matud, 2004; McDonough \& Walters, 2001), women in our sample reported higher levels of perceived stress than men, confirming our first hypothesis. In their meta-analysis, Tamres, Janicki, and Helgeson (2002) reported that none of the included studies found that men reported higher 
levels of stress than women. However, it is still unclear whether this difference is due to actually existing differences or to gender norms. Studies suggest that women may be more willing to admit to feeling stressed whereas men may see this as showing weakness and refrain from reporting actual levels (Matheny, Ashby, \& Cupp, 2005). To help resolve this question, future stress evaluations should not only rely on self-reports, but also utilize other methods, such as direct observations or measurements of stress hormones.

Table 6

Stage distribution and perceived stress and coping across stages of change $(n=1,356)$

\begin{tabular}{|l|c|c|c|c|c|c|}
\hline & & Precontemplation & Contemplation & Preparation & Action & Maintenance \\
\hline \multirow{2}{*}{ Male } & $\mathrm{n}$ & 172 & 33 & 34 & 105 & 257 \\
& $\%$ & 28.6 & 5.5 & 5.7 & 17.5 & 42.8 \\
\hline \multirow{2}{*}{ Female } & $\mathrm{n}$ & 177 & 32 & 37 & 128 & 381 \\
& $\%$ & 23.4 & 4.2 & 4.9 & 17.0 & 50.5 \\
\hline Perceived stress & $\mathrm{M}$ & 2.07 & 2.31 & 2.30 & 2.31 & 2.44 \\
level & $\mathrm{SD})$ & $(1.15)$ & $(1.13)$ & $(1.06)$ & $(1.16)$ & $(1.08)$ \\
\hline \multirow{2}{*}{$\begin{array}{l}\text { Perceived coping } \\
\text { effectiveness }\end{array}$} & $\mathrm{M}$ & 3.50 & 3.06 & 2.97 & 3.35 & 3.64 \\
& $\mathrm{SD})$ & $(1.08)$ & $(0.98)$ & $(1.15)$ & $(1.03)$ & $(0.90)$ \\
\hline
\end{tabular}

On a subscale level, we only found small gender differences for personal stressors. This scale also includes work-related items and on a single-item level, especially these items showed differences between men and women. Men were more likely to be stressed by work, unemployment, living situation, and conflict with friends and work, whereas women were more likely to be stressed by the health of family and friends. These results support our second hypothesis. Matud (2004) also reported that men were more likely to be stressed by work and relationships with friends. However, the effect size was small in that study as it was in our study. Observing that effect sizes were small in other studies, Matud (2004) attributes this pattern to social changes in gender roles and constraints. Gender roles are increasingly being shared as more women step into the workplace and more men take care of the household and childcare matters, which may contribute to the small effect size.

While there was no difference in perceived coping effectiveness, our third hypothesis was supported with our results showing significant gender difference in coping strategies. More women reported using adaptive coping strategies while men were more likely to use maladaptive and avoidance strategies. Women were more likely than men to eat, pray, and talk to friends and families to cope with stress while men were more likely to drink, smoke, take drugs, or ignore the problem when faced with a stressful situation. Matheny, Ashby, and Cupp (2005) also found that women used more adaptive coping strategies than men. However, despite having better coping resources than males, they found that women were more likely to report illnesses. This suggests that stress might manifest itself differently in men and women. Women might be more likely to develop depressive or anxiety disorders when overwhelmed with stress, while men may be more prone to developing substance abuse problems or physical illnesses (Andre-Petersson, Hedblad, Janzon, \& Ostergren, 2006). Although our study did not measure health outcomes directly related to stress, this might be an important issue to explore in future studies. Other studies have reported that men are more likely to engage in instrumental coping strategies, such as actively fighting the source of stress, which in turn is associated with better health. Inconsistencies in findings may be due to 
the fact that coping is rarely measured in the context of the stressful situations (McDonough $\&$ Walters, 2001). The use of different coping methods may depend on the situation. For example, if a stressor in the workplace cannot be changed, it may be more productive to use avoidance or distraction strategies, than to actively fight that cause.

As had been reported in previous studies (Padlina et al., 2001; Keller et al., 2002) we found no significant differences in the distribution across the stages of stress management between men and women which confirmed our fourth hypothesis. Assessing the population's readiness for behavior change can provide important information about the population's receptiveness for future interventions. In our sample, we found that most people were in maintenance stage of the TTM, while about one-third were in precontemplation. Interestingly, very few people were in contemplation or preparation stages. Studies have found that those in the maintenance stage were less likely to have symptoms of illness, illustrating the importance of developing effective stress management interventions (Padlina et al., 2001). Evers et al. (2006) have shown in a first study that tailoring interventions to the individuals' readiness for stress management can result in favorable outcomes. Individuals in the precontemplation stage reported the lowest levels of perceived stress and one of the highest levels of perceived coping effectiveness. This indicates that a large number of individuals in this group think they do not have much stress and do not need to do anything beyond their already effective strategies. While individuals in the maintenance stage show the highest level of perceived stress, they also show the highest score for coping effectiveness. For stage tailored stress management interventions, an effort should be made to identify especially individuals with high perceived stress and low perceived coping effectiveness.

Our findings were affected by some methodological limitations that may affect the generalizability of our results. Because the HHI survey is a large cross sectional survey which measured a wide range of health behaviors, questions on stress were limited to a total of 13 questions on stressors and 10 questions on coping strategies. Other questionnaires that focus solely on stress are usually much more detailed and in-depth. For example, the Coping Resources Inventory for Stress (CRIS) is a 220item inventory that measures coping strategies (Matheny, Aycock, Curlette, \& Junker, 1993). Furthermore, defining coping strategies is a complex issue that studies have not been able to standardize (Tamres, Janicki \& Helgeson, 2002). Some studies categorize coping strategies as problem-focused versus emotion-focused while others divide coping strategies as adaptive or maladaptive.

The survey was conducted over the phone, which excluded those who did not have landlines, declined to answer survey questions, were not at home at the time the surveyor called, or those who did not have a permanent residence. In addition, this survey was an English-language-only survey, which excluded over 70,000 non-English speaking residents statewide (US Department of Labor, 2000). The participants in this survey were in a higher income bracket compared to the state's average income level. Studies show that education and income levels have a negative impact on overall health status, indicating that our sample may be healthier than the general Hawaii population (Prus, 2007). In addition, those who are more stressed may have declined to participate in the survey, thereby increasing our sampling error. This survey also relied on self-reports, which may have caused participants to give socially desirable or acceptable answers. For example, women may have felt more comfortable and willing to report their levels of stress whereas men may have felt restricted by gender norms to admit to feeling stressed (Tamres, Janicki, \& Helgeson, 2002).

One of the strengths of our study was that it was the first statewide survey with a large sample size that analyzed gender differences in perceived stress and coping strategies in Hawaii. Our sample was also representative of Hawaii's diverse ethnic population and included residents of all inhabited Hawaiian Islands. The overall quality of our short screening measures was 
satisfactory. The medium high to low scale inter-correlations pointed to the validity of the measures with few redundant variables. In addition, this survey was one of the few surveys that examined the stages of readiness for stress management.This information is essential in planning future research and future stress intervention programs for Hawaii's populations.
Since the experience of stress and the strategies to cope with stress are determined by multiple factors, future studies should focus on additional variables like age, ethnicity, acculturation, social support, and environmental conditions in order to adequately plan potentially effective intervention programs for Hawaii's diverse populations.

\section{References}

Andre-Petersson, L., Hedblad, B., Janzon, L., \& Ostergren, P. O. (2006). Social support and behavior in a stressful situation in relation to myocardial infarction and mortality: Who is at risk? Results from prospective cohort study "Men born in 1914," Malmo, Sweden. International Journal of Behavioral Medicine, 13, 340-347.

Cohen, J. (1988). Statistical power analysis for the behavioral sciences (2nd ed.). Hillsdale, NJ: Erlbaum.

Everly, G. S., \& Lating, J. M. (Eds.). (2002). A clinical guide to the treatment of the human stress response (2nd ed.). New York: Plenum Publishers.

Evers, K. E., Prochaska, J. O., Johnson, J. L., Mauriello, L. M., Padula, J. A., \& Prochaska, J. M. (2006). A randomized clinical trial of a population- and transtheoretical model-based stress management intervention. Health Psychology, 25, 521-529.

Gore-Felton, C., Koopman, C., Spiegel, D., Vosvick, M., Brodino, M., \& Winningham, A. (2006). Effects of quality of life and coping in depression among adults living with HIV/AIDS. Journal of Health Psychology, 11, 711-729.

Greenglass, E. R. (1995). Gender, work stress, and coping: Theoretical implications. Journal of Social Behavior and Personality, 10, 121-134.

Hanna, J. M. (1998). Migration and acculturation among Samoans: Some sources of stress and support. Social Science Medicine, 46, 1325-1336.

Harriman, L. C. (1983). Personal and marital changes accompanying parenthood. Family Relations, 32, 387-394.

Hawaii State Government. (2004). Department of Business, Economic Development, \& Tourism, 2004 Hawaii State data book, section 14, table 14.11. Retrieved April 23, 2007, from http://www.Hawaii.gov/dbedt/info/economic/databook/db2004/

Hogan, J. M., Carlson, J. G., \& Dua, J. (2002). Stressors and stress reactions among university personnel. International Journal of Stress Management, 9, 289-310.

Keller, S., Kaluza, G., Baum, E. \& Basler, H. D. (2002). Getting ready to relax. International Journal of Behavioral Medicine, 9, 142.

Kim, U., Yang, K., \& Hwang, K. (Eds.). (2006). Indigenous and cultural psychology: Understanding people in context. New York: Springer Sciences \& Business Media, Inc.

Lindquist, T. L., Beilin, L. J., \& Knuiman, M. W. (1997). Influence of lifestyle, coping, and job stress on blood pressure in men and women. Hypertension, 29, 1-7.

Makosky, V.P. (1980). Stress and the mental health of women. In M. Guttentag, S. Salasin, \& D. Belle (Eds.), The mental health of women (pp. 111-127). New York: Freeman Press.

Matheny, K. B., Aycock, D. W., Curelette, W. L., Junker, G. N. (1993). The coping resources inventory for stress: A measure of perceived resourcefulness. Journal of Clinical Psychology, 49, 815-830

Matheny, K. B., Ashby, J. S., \& Cupp, P. (2005). Gender difference in stress, coping, and illness among college students. The Journal of Individual Psychology, 61, 365-379.

Matud, P.M. (2004). Gender differences in stress and coping style. Personality and Individual Differences, 37, 1401-1415.

McDonough, P. \& Walters, V. (2001). Gender and health: Reassessing patterns and explanations. Social Science and Medicine, 52, 547-559. 
National Institute of Child Health and Human Development. (2007). National Institute of Health. Stress system malfunction could lead to serious, life threatening disease. Retrieved April 1, 2007, from http://www.nih.gov/news/pr/sep2002/nichd-09.htm

O'Hea, E. L., Wood, K. B., \& Brantley, P. J. (2003). The Transtheoretical Model: Gender differences across 3 health behaviors. American Journal of Health Behavior, 27, 645-656.

Padlina, O., Aubert, L., Gehring, T. M., Martin-Diener, E., \& Somaini, B. (2001). Stages of change for perceived stress in a Swiss population sample: An explorative study. Spzial-und Praventivmedizin, 46, 396-403.

Plaisier, I., de Bruijn, J. G., de Graaf, R., ten Have, M., Beekman, A. T., \& Penninx, B. W. (2007). The contribution of working conditions and social support to the onset of depressive and anxiety disorders among male and female employees. Social Science \& Medicine, 64, 401-410.

Prochaska, J. O., \& Velicer, W.F. (1997). The Transtheoretical Model of health behavior change. American Journal of Health Promotion, 12, 38-48.

Prus, S. G. (2007). Age, SES, and health: A population level analysis of health inequalities over the lifecourse. Sociology of Health and Illness, 29, 275-296.

Ptacek, J. T., Smith, R. E., \& Zanas, J. (1992). Gender, appraisal, and coping: A longitudinal analysis, Journal of Personality, 60, 477.

SPSS, Inc. (2005). SPSS for Windows, rel. 15.0.0. Chicago: Author.

Tamres, L. K., Janicki, D., \& Helgeson, V. S. (2002). Sex differences in coping behavior: A meta-analytic review and an examination of relative coping. Personality and Social Psychology Review, 6, 230.

Torkelson, E., \& Muhonen, T. (2004). The role of gender and job level in coping with occupational stress. Work \& Stress, 18, 267-274.

Tyler, P. A., \& Ellison, R. N. (1994). Sources of stress and psychological well-being in high dependency nursing. Journal of Advanced Nursing, 19, 469-476.

Tytherleigh, M. Y, Jacobs, P. A., Webb, C., Ricketts, C., \& Cooper, C. (2007). Gender, health, and stress in English University staff-exposure or vulnerability? Applied Psychology: An International Review, 56, 267-287.

United States Census Bureau. (2005). State and county quick fact. Retrieved April 27, 2007, from http://quickfacts.census.gov/gfd/states/15/15003.html

United States Department of Labor. (2000). 2000 Census of Population and Housing. Retrieved May 3, 2007, from http://www.census.gov/prod/cen2000/doc/ProfilesTD.pdf

Wang, J., \& Patten, S. B. (2002). The moderating effects of coping strategies on major depression in the general population. Canadian Journal of Psychiatry, 47, 167-173.

Weidner, G. (2000). Why do men get more heart disease than women? An international perspective. Journal of American College of Health, 48, 291-294.

\section{Acknowledgements}

This study was funded by the Hawaii Tobacco Settlement Special Fund through a contract from the Hawaii Department of Health to Dr. Maddock.

\section{Author Information}

Lauren A. Gentry, B.A.

University of Hawaii at Manoa

Department of Public Health Sciences, School of Medicine

1960 East-West Rd.

Honolulu, HI 96822

E-Mail: gentry@hawaii.edu 
Jane J. Chung, B.A.

University of Hawaii at Manoa

Department of Public Health Sciences, School of Medicine

1960 East West Road

Honolulu, HI 96822

E-Mail: chungjae@hawaii.edu

Nandar Aung, M.P.H.

University of Hawaii at Manoa

Department of Public Health Sciences, School of Medicine 1960 East West Road

Honolulu, HI 96822

E-Mail: andar@hawaii.edu

Stefan Keller, Ph.D.*

University of Hawaii at Manoa

Department of Public Health Sciences, School of Medicine 1960 East West Road, Biomed D104

Honolulu, HI 96822

Ph.: 1-808-956-4553

Fax.:1-808-956-5818

E-Mail: kellers@hawaii.edu

Katie M. Heinrich, Ph.D.

University of Hawaii at Manoa

Department of Public Health Sciences, School of Medicine 1960 East West Road, Biomed D104

Honolulu, HI 96822

E-Mail: katiemh@hawaii.edu

Jay E. Maddock, Ph.D.

University of Hawaii at Manoa

Department of Public Health Sciences, School of Medicine 1960 East West Road, Biomed D204

Honolulu, HI 96822

E-Mail: jmaddock@hawaii.edu

* corresponding author 